\title{
Manganese uptake in marine bacteria; the novel MntX transporter is widespread in Roseobacters, Vibrios, Alteromonadales and the SAR11 and SAR116 clades
}

\author{
Robert T Green ${ }^{1,2}$, Jonathan D Todd ${ }^{1}$ and Andrew WB Johnston ${ }^{1}$ \\ ${ }^{1}$ School of Biological Sciences, University of East Anglia, Norwich Research Park, Norwich, \\ UK and ${ }^{2}$ Institute of Food Research, Colney Lane, Norwich Research Park, Norwich, UK
}

\begin{abstract}
We showed that two very different manganese transporters occur in various important genera of marine bacteria. The ABC transporter encoded by sitABCD of the model Roseobacter-clade bacterium Ruegeria pomeroyi DSS-3 is required for $\mathrm{Mn}^{2+}$ import and was repressed by the Mur (Manganese uptake regulator) transcriptional regulator in Mn-replete media. Most genomesequenced Roseobacter strains contain SitABCD, which are in at least two sub-groups, judged by their amino-acid sequences. However, a few Roseobacters, for example, Roseovarius nubinhibens, lack sitABCD, but these contain another gene, $m n t X$, which encodes a predicted inner membrane polypeptide and is preceded by cis-acting Mur-responsive MRS sequences. It was confirmed directly that $m n t X$ of Roseovarius nubinhibens encodes a manganese transporter that was required for growth in Mn-depleted media and that its expression was repressed by Mur in Mn-replete conditions. MntX homologues occur in the deduced proteomes of several bacterial species. Strikingly, all of these live in marine habitats, but are in distantly related taxonomic groups, in the $\gamma$ - and $\alpha$ proteobacteria. Notably, MntX was prevalent in nearly all strains of Vibrionales, including the important pathogen, Vibrio cholerae. It also occurs in a strain of the hugely abundant Candidatus Pelagibacter (SAR11), and in another populous marine bacterium, Candidatus Puniceispirillum marinum (SAR116). Consistent with this, MntX was abundant in marine bacterial metagenomes, with one sub-type occurring in an as-yet unknown bacterial clade.

The ISME Journal (2013) 7, 581-591; doi:10.1038/ismej.2012.140; published online 29 November 2012

Subject Category: geomicrobiology and microbial contributions to geochemical cycles

Keywords: manganese; MntX; Mur; regulation; Roseobacters; SAR11; SAR116; SitABCD; Vibrio
\end{abstract}

\section{Introduction}

Manganese $(\mathrm{Mn})$ is an essential metal, being found in a wide range of enzymes, particularly those involving redox chemistry, as well as being a critical component of the photosystem II in plants, algae and cyanobacteria (Wieghardt, 1989). Although abundant in the Earth's crust, it is scarce in the oceans, reaching only low nanomolar concentrations in near-surface waters (Landing and Bruland, 1987; Middag et al., 2011). Marine manganese can take the form of insoluble oxides $\left(\mathrm{Mn}^{3+}\right.$ and $\left.\mathrm{Mn}^{4+}\right)$, as well as the soluble, $\mathrm{Mn}^{2+}$ version, which can arise via photochemical reduction of $\mathrm{Mn}$ oxides (Sunda and Huntsman, 1994). Conversely, several bacteria contain enzymes that oxidise Mn(II) (Tebo

Correspondence: A Johnston, School of Biological Sciences, University of East Anglia, Norwich Research Park, Norwich NR4 7TJ, UK.

E-mail: a.johnston@uea.ac.uk

Received 25 August 2012; revised 1 October 2012; accepted 1 October 2012; published online 29 November 2012 et al., 2005); such strains include some Roseobacters (see below) and other classes of marine bacteria.

Studies on manganese import in a range of different terrestrial bacteria have revealed two, widely used, but very different, specialised $\mathrm{Mn}^{2+}$ transporters, termed MntH and SitABCD, with some bacteria (for example, Salmonella), harbouring both systems (Boyer et al., 2002). MntH is an inner membrane symporter that resembles the Nramp proteins of eukaryotes (Makui et al., 2000). In contrast, SitABCD is an ABC-transporter type system, initially thought to be involved in iron uptake hence its somewhat misleading acronym of Salmonella iron transporter (Zhou et al., 1999). Recently, Hohle et al. (2011) described a novel ion channel polypeptide, MnoP, which facilitates $\mathrm{Mn}^{2+}$ transport across the outer membrane of $B$. japonicum, the microsymbiont of soybeans: this polypeptide is confined to a few close relatives of Bradyrhizobium.

Bacteria are known to use two different, dedicated, Mn-responsive transcriptional regulators, termed MntR and Mur (Manganese uptake 
regulator). The former controls manganese homoeostasis in Escherichia coli, regulating the expression of four genes, including the $\mathrm{MntH}$ transporter, by Mn-dependent binding to their operator sequences in Mn-replete cells (Yamamoto et al., 2011). Bioinformatic analyses strongly indicated that a few $\alpha$-proteobacterial species also contain MntR, repressing expression of their cognate $\mathrm{MntH}$ or SitABCD transporters in response to Mn availability (Rodionov et al., 2006). However, the manganese regulons of $\alpha$-proteobacterial species that have been examined directly, in rhizobia and the mammalian pathogen Brucella, are controlled by a very different transcriptional regulator, Mur. When complexed with $\mathrm{Mn}^{2+}$, Mur represses transcription by binding to cis-acting 'Mur-responsive sequences' (MRSs), located at the Mn-repressed promoters of the sit $A B C D$ transporter operon and of promoters that precede other $\mathrm{Mn}$ regulated genes (Chao et al., 2004; Díaz-Mireles et al., 2004, 2005; Platero et al., 2007; Hohle et al., 2011; Menscher et al., 2012). The Mur protein resembles the iron responsive regulator Fur (ferric uptake regulator), which controls iron homoeostasis in many different bacteria (Lee and Helmann, 2007). In the $\alpha$ proteobacteria, the Mur version of the Fur superfamily likely evolved from Fur, sensu stricto, but responds to a different, although similar, metal and regulates only a few transcriptional units. In turn, this may be because bacteria in this sub-phylum no longer use Fur for iron homoeostasis; instead, they employ evolutionarily distinct transcriptional regulators like Irr and, in some lineages, RirA (Rudolph et al., 2006; Johnston et al., 2007).

To date, there are no direct studies on the mechanisms of $\mathrm{Mn}$ acquisition or Mn-responsive gene regulation in any marine microbes. Among the most populous, widespread classes of marine bacteria in the near-surface waters of the World's oceans are the Roseobacters, a taxonomically coherent group of $>25$ genera within the Rhodobacterales Family of $\alpha$-proteobacteria ('Roseobase' at http:// www.roseobase.org/; Buchan et al., 2005; Brinkhoff et al., 2008). The acquisition and the responses of these bacteria to various nutrients, including trace metals, will therefore have important effects on the corresponding biogeochemical cycles in the oceans.

The first Roseobacter whose genome was sequenced is Ruegeria pomeroyi DSS-3 (Moran et al., 2004), which is something of a reference strain for genetic and genomic studies on this clade (Moran et al., 2012; Curson et al., 2011). This strain has a homologue of the Mur regulator and its genome includes an operon that likely encodes a SitABCD-type $\mathrm{Mn}^{2+}$ transporter, but it lacks $\mathrm{MntH}$ and has no homologue of the MntR transcriptional regulator (Rodionov et al., 2006). Here, we describe genetic analyses of $\mathrm{Mn}^{2+}$ uptake and its regulation in Ruegeria pomeroyi DSS-3. Furthermore, comparative genomic bioinformatic analyses of other Roseobacters uncovered a novel $\mathrm{Mn}^{2+}$ transporter, which occurs in a range of important marine bacteria and is widespread in marine microbial metagenomes.

\section{Materials and methods}

Strains, media and growth conditions

Ruegeria pomeroyi wild-type DSS-3 (Moran et al., 2004) was used as the source of genomic DNA for PCR amplifications. A Rif ${ }^{\mathrm{R}}$ derivative, J470 (Todd et al., 2011), was the recipient for plasmids in triparental conjugational matings, using the helper plasmid pRK2013 (Figurski and Helinski, 1979). Strain 803 (Wood, 1966) was the routine E. coli host.

Ruegeria pomeroyi was grown at $28^{\circ} \mathrm{C}$ in complete half-strength YTSS medium (González et al., 2003), or in minimal RSS medium before $\beta$-galactosidase assays (Rossen et al., 1985). RSS minimal medium was based on the marine basal medium of Baumann and Baumann (1981), but the 'sea salts' was replaced with $\left(\mathrm{l}^{-1}\right)$ : $17 \mathrm{~g} \mathrm{NaCl}$, $1.5 \mathrm{~g}^{\mathrm{MgCl}}{ }_{2}$, $0.75 \mathrm{~g} \mathrm{CaCl}_{2}, 0.75 \mathrm{~g} \mathrm{KCl}, 20 \mu \mathrm{M} \mathrm{FeCl}_{3}$, plus the vitamin solution of González et al. (1997). Where appropriate, $\mathrm{MnCl}_{2}$ was added, to $20 \mu \mathrm{M}$.

\section{DNA manipulations and plasmid constructions}

Routine manipulations of DNA were as in Wexler et al. (2001).

\section{Construction of recombinant plasmids and mutants} The approach used to make insertions into sit $A$ and mur involved the cloning of fragments internal to each of these genes into a suicide plasmid that was transferred by conjugation into Ruegeria pomeroyi, selecting for mutants in which the plasmid had integrated into the corresponding, genomic version of the gene (see Supplementary information, which also describes how the lacZ transcriptional fusions and other recombinant plasmids were made).

The sequences of the primers that were used for the various manipulations are shown in Supplementary Table S3.

\section{Results}

Ruegeria pomeroyi DSS-3 sitABCD is required for $\mathrm{Mn}^{2+}$ import and is regulated by Mur

Inspection of the Ruegeria pomeroyi DSS-3 genome had revealed two sets of genes with a likely role in $\mathrm{Mn}$ uptake and in regulating this process (Rodionov et al., 2006). The consecutive genes SPO3366, SPO3365, SPO3364 and SPO3363 are predicted to encode the ABC-class $\mathrm{Mn}^{2+}$ transporter SitABCD (Figure 1a); sit $A$ encodes the periplasmic binding protein, sitB the ATPase and sitC and sitD the two inner membrane permease polypeptides. The other relevant gene is SPO2477, a single-gene transcriptional unit whose product is $67 \%$ identical to Mur of 


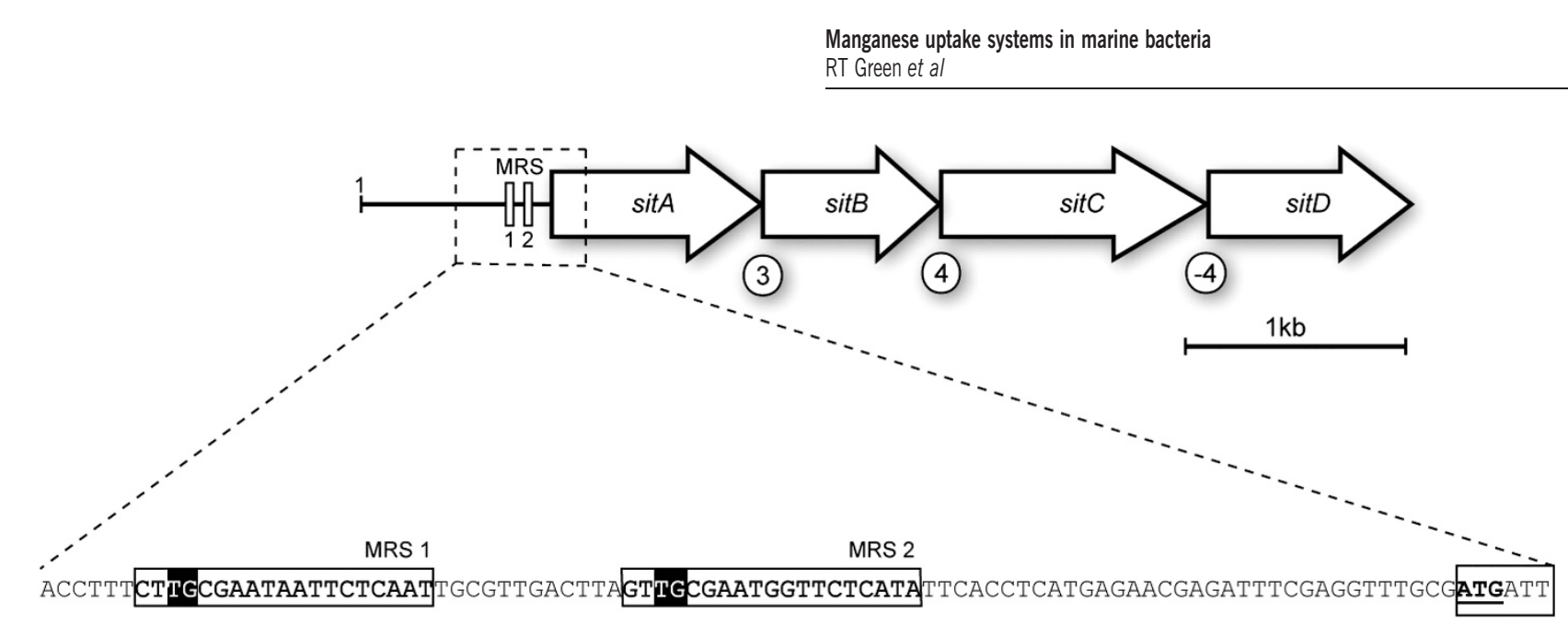

sitA

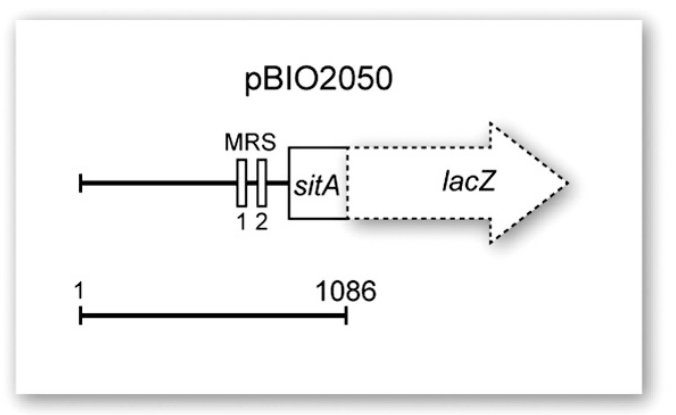

Figure 1 Maps of sitABCD genes of Ruegeria pomeroyi and their genetically modified derivatives. The open arrows in the top section show the dimensions of the individual genes in the sitABCD operon, the corresponding gene tags being SPO3366, SPO3365, SPO3364 and SPO3363, respectively. Numbers of base pairs in intergenic spaces are shown, ' -4 ' denoting overlap between sitC and sitD. The approximate locations of the two MRS regulatory sequences are indicated. The middle section shows the sequence upstream of the ATG start of sitA, the two MRS motifs being boxed. The conserved 'TG' base pairs in MRS1 and in MRS2 that were changed by site-directed mutagenesis are highlighted. The dimensions of the 1086-bp fragment used to construct the sitA-lac $Z$ transcriptional fusion plasmid pBIO2050 are shown.

Rhizobium leguminosarum, which represses expression of sit $A B C D$ in that species. We confirmed that Ruegeria pomeroyi sitABCD and mur are indeed involved in Mn uptake as follows.

First, an insertional mutation was made into sit $A$ (Supplementary information) and growth of the resultant mutant, strain J529, was compared with the wild type in minimal media that were replete or depleted for manganese. The two strains grew at similar rates in media supplemented with $20 \mu \mathrm{M}$ $\mathrm{MnCl}_{2}$, but in the absence of added manganese, the sitA mutant was severely reduced in its growth rate (Figure 2). Thus Ruegeria pomeroyi SitABCD is its major, and likely its only dedicated Mn transporter, with the slight growth of the mutant that is eventually seen in the Mn-depleted medium, perhaps being due to nonspecific import of the metal by other transporters.

To assess if sitABCD expression was affected by $\mathrm{Mn}^{2+}$ availability, we made a transcriptional fusion plasmid, pBIO2050, in which the lacZ reporter of the wide host-range promoter-probe vector pBIO1878 was controlled by the sitA promoter (Figure 1). This fusion plasmid was mobilised into wild-type Ruegeria pomeroyi by conjugation, and a purified transconjugant was grown in Mn-replete and Mn-depleted minimal media, before assaying $\beta$ galactosidase, encoded by the lac $Z$ reporter. There was markedly greater (19-fold) expression in the - Mn than in + Mn medium (Table 1).

This differential expression was shown to be mediated by the Mur repressor by repeating this assay, but this time, the fusion plasmid pBIO2050 was transferred into a Mur- mutant (J528), which had an insertion in the mur gene, SPO2477. In this background, the sitA-lacZ fusion expressed $\beta$-galactosidase at high, constitutive levels in both - Mn and + Mn media (Table 1).

Just as in the region upstream of sitABCD of Rhizobium leguminosarum (Díaz-Mireles et al., 2004), there are two motifs, centred 75 and $45 \mathrm{bp}$ upstream of the ATG translational start of Ruegeria pomeroyi sitA, both of which resemble a regulatory 'MRS-box' (Rodionov et al., 2006); these were termed MRS1 and MRS2, respectively (Figure 1). These cis-acting sequences bind to Mur and are required for Mur-dependent repression (Rodionov et al., 2006). To investigate their roles in Ruegeria, we made site-directed mutations in each of them individually, and also in both MRSs, substituting their highly conserved GT residues with two A residues. We cloned the resultant mutant fragments 

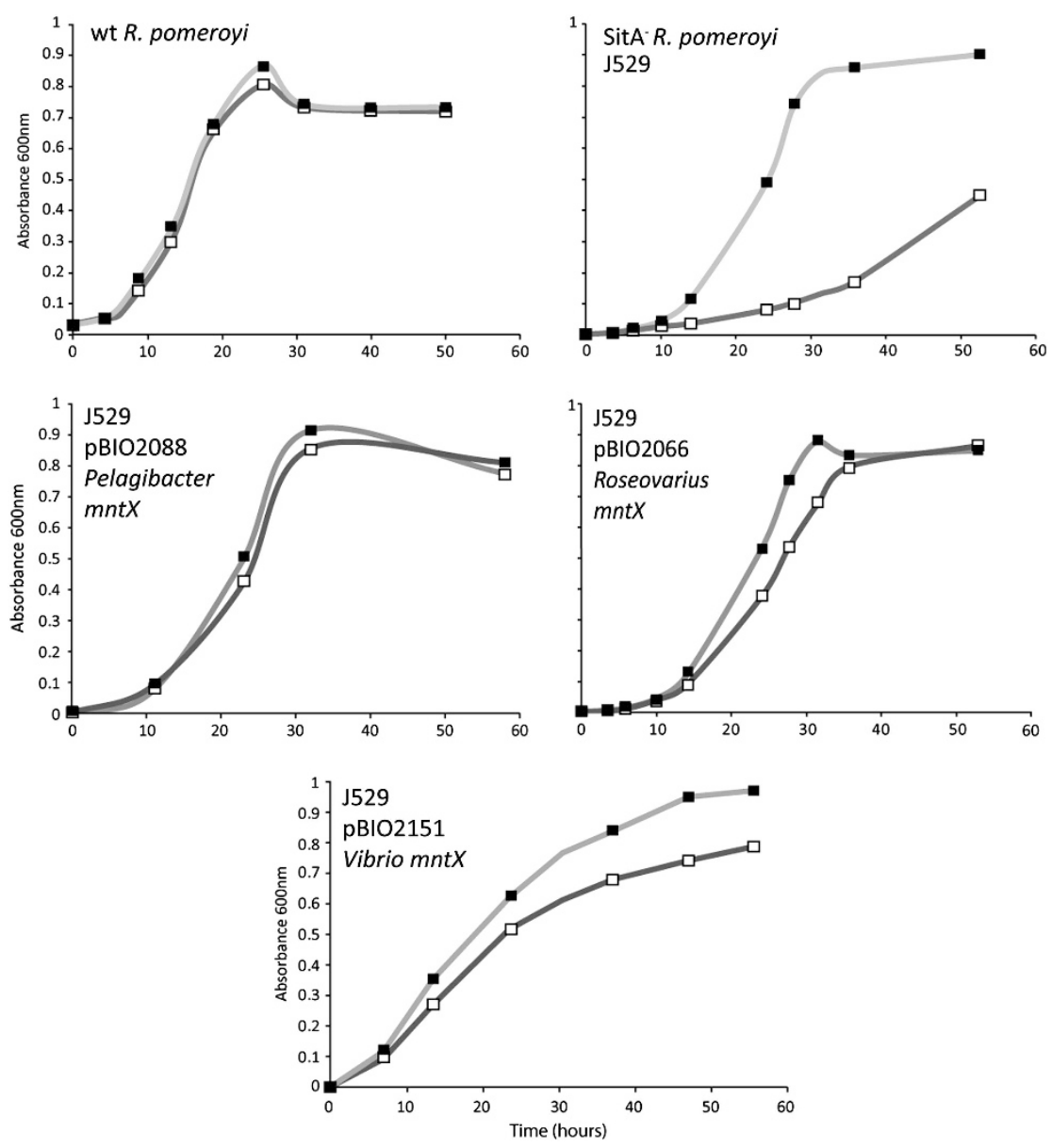

Figure 2 Effects of sitA and of the mntX genes of Roseovarius nubinhibens, Ca. Pelagibacter ubique and Vibrio cholerae on growth of Ruegeria pomeroyi in Mn-depleted minimal media. Cultures of Ruegeria pomeroyi wild type, or the SitA- mutant J529 or J529 corrected with cloned mntX of Roseovarius nubinhibens (pBIO2066), Ca. Pelagibacter ubique (pBIO2088) or V. cholerae (pBIO2150) were diluted into RSS minimal medium either lacking any added $\mathrm{MnCl}_{2}$ (white squares), or supplemented with $10 \mu \mathrm{M} \mathrm{MnCl}_{2}$ (black squares). Cultures were grown, with shaking, at $28^{\circ} \mathrm{C}$, and growth was measured by absorbance at $600 \mathrm{~nm}$.

Table 1 The MRS motif and the mur genotype affect manganese-responsive expression of a Ruegeria pomeroyi sitA-lacZ transcriptional fusion.

\begin{tabular}{lccccc}
\hline & WT (sitA-lacZ) & Mur (sitA-lacZ) & $\mu M R S 1$ (sitA-lacZ) & $\mu M R S 2$ (sitA-lacZ) & $\mu M R S 1+\mu M R S 2($ sitA-lacZ) \\
\hline Plus Mn & $42 \pm 1$ & $1736 \pm 178$ & $294 \pm 14$ & $249 \pm 5$ & $1612 \pm 41$ \\
Minus Mn & $791 \pm 30$ & $1817 \pm 204$ & $628 \pm 19$ & $927 \pm 23$ & $1235 \pm 98$ \\
\hline
\end{tabular}

The wild type sitA-lacZ fusion plasmid pBIO2050, or derivatives (pBIO2100, pBIO2101 and pBIO2102, respectively) containing mutations in the MRS1 ( $\mu$ MRS1) or MRS2 ( $\mu$ MRS1) or in both ( $\mu$ MRS1 + $\mu$ MRS2) regulatory sequences were introduced into wild-type and Mur mutant Ruegeria pomeroyi DSS-3 recipients. Transconjugants were grown in media that either did or did not have added $\mathrm{MnCl}_{2}$ before assaying $\beta$-galactosidase in triplicate. Activities are shown in Miller units, with s.e.

into the reporter plasmid pBIO1878. These mutated plasmids were each introduced by conjugation into wild-type Ruegeria pomeroyi and assayed for $\beta$ galactosidase activity. Compared with the wild-type fusion, the mutation in the upstream MRS1 caused a $\sim$ seven-fold increase in $\beta$-galactosidase activity in Mn-replete conditions, but was little changed under Mn-limitation. Similarly, the mutation in MRS2 caused a six-fold higher expression of $\beta$-galactosidase than the wild type in Mn-replete conditions (Table 1). The fusion plasmid with mutations in both MRS1 and MRS2 was deregulated even further, under both Mn replete (38-fold) and Mn-depleted (1.5-fold) conditions. Thus, both MRS1 and MRS2 contribute to the Mn-responsive, Mur-dependent repression of the sit $A B C D$ operon.

Diversity of the mur, sitABCD and other genes involved in Mn uptake and sensing in the Roseobacters Inspection of the genomes of 37 other Roseobacters showed that none of them contained the MntR 
Table 2 Distribution of sub-types of the sitA, $m n t X, m n t H$ and mur genes in genome-sequenced Roseobacter strains

\begin{tabular}{|c|c|c|c|c|}
\hline Species & $\begin{array}{l}\text { U్ } \\
\frac{\infty}{5} \\
\frac{5}{n}\end{array}$ & $\underset{\text { È }}{\stackrel{x}{E}}$ & $\underset{\text { है }}{\stackrel{T}{\Sigma}}$ & ई \\
\hline \multicolumn{5}{|l|}{ Citreicella SE45 } \\
\hline Citreicella sp. 357 & & & & 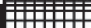 \\
\hline \multicolumn{5}{|c|}{ Dinoroseobacter shibae DFL 12} \\
\hline \multicolumn{5}{|l|}{ Jannaschia sp. CCS1 } \\
\hline \multicolumn{5}{|c|}{ Loktanella vestfoldensis SKA53 } \\
\hline \multicolumn{5}{|c|}{ Maritimibacter alkaliphilus HTCC2654 } \\
\hline \multirow{2}{*}{\multicolumn{5}{|c|}{$\begin{array}{c}\text { Oceanibulbus indolifex HEL45 } \\
\text { Oceanicola batsensis HTCC2597 }\end{array}$}} \\
\hline & & & & \\
\hline \multicolumn{5}{|c|}{ Oceanicola granulosus HTCC2516 } \\
\hline \multicolumn{5}{|c|}{ Octadecabacter antarcticus 307} \\
\hline \multicolumn{5}{|c|}{ Octadecabacter arcticus 238} \\
\hline \multicolumn{5}{|c|}{ Pelagibaca bermudensis HTCC2601 } \\
\hline \multicolumn{5}{|c|}{ Phaeobacter gallaeciensis 2.10} \\
\hline \multicolumn{5}{|c|}{ Phaeobacter gallaeciensis BS107 } \\
\hline \multicolumn{5}{|l|}{ Phaeobacter sp. Y4I } \\
\hline \multicolumn{5}{|c|}{ Rhodobacterales bacterium HTCC2083 } \\
\hline \multicolumn{5}{|c|}{ Rhodobacterales bacterium HTCC2150 } \\
\hline \multicolumn{5}{|c|}{ Rhodobacterales bacterium HTCC2255 } \\
\hline \multicolumn{5}{|c|}{ Rhodobacterales bacterium KLH11 } \\
\hline \multirow{2}{*}{\multicolumn{5}{|c|}{ Roseobacter denitrificans OCh 114}} \\
\hline \multirow{2}{*}{\multicolumn{5}{|c|}{$\begin{array}{c}\text { Roseobacter litoralis Och } 149 \\
\text { Roseobacter } \mathrm{sp} . \text { AzwK-3b }\end{array}$}} \\
\hline & & & & \\
\hline \multicolumn{5}{|l|}{ Roseobacter sp. CCS2 } \\
\hline \multicolumn{5}{|l|}{ Roseobacter sp. GAl101 } \\
\hline \multicolumn{5}{|l|}{ Roseobacter sp. MED193 } \\
\hline \multicolumn{5}{|l|}{ Roseobacter sp. SK209-2-6 } \\
\hline \multicolumn{5}{|l|}{ Roseovarius nubinhibens ISM } \\
\hline \multicolumn{5}{|l|}{ Roseovarius sp. 217} \\
\hline \multicolumn{5}{|l|}{ Roseovarius sp. TM1035 } \\
\hline \multicolumn{5}{|c|}{ Ruegeria lacuscaerulensis ITI-1157 } \\
\hline Ruegeria pomeroyi DSS- & & & & \\
\hline Ruegeria sp. R11 & & & & \\
\hline Ruegeria sp. TM1040 & & & & \\
\hline Ruegeria sp. Trich CH4B & & & & \\
\hline Sagittula stellata $\mathrm{E}-37$ & & & & \\
\hline Sulfitobacter NAS-14.1 & & & & \\
\hline Sulfitobacter sp. EE-36 & & & & \\
\hline Thalassiobium R2A62 & & & & \\
\hline
\end{tabular}

In-fills with the same shading patterns represent polypeptides that closely resemble each other. In the case of the SitA, B, C and D polypeptides, all four components of this transport system showed a coherent relatedness pattern; thus all four polypeptides of (e.g.) Sulfitobacter were most similar to those in strains (e.g. Octadecabacter) with the same in-fill pattern. Empty boxes represent absence of the corresponding polypeptide. Note that $O$. antarcticus 307 has two Mur sequences, one resembling that in most of the Roseobacters (including Ruegeria pomeroyi) and one that is similar to those in Sulfitobacter, Pelagibaca and Roseobacter GAI101.

transcriptional regulator, but that all of them contained at least one gene whose product was within the Mur branch of the Fur super-family. However, we discerned different sub-types of these Mur polypeptides, judged by their sequences in different strains and species (Table 2; Supplementary Figure S1). In most Roseobacter strains, the Mur polypeptide closely resembles ( $>70 \%$ identical) that of Ruegeria pomeroyi DSS-3 (above). However, those of Sulfitobacter, Citreicella, Pelagibaca, and one strain (GAI101) of Roseobacter are only $\sim 40 \%$ identical to that of Ruegeria pomeroyi, with those of Sulfitobacter, Pelagibaca bermudensis and Roseobacter GAI101 being very similar to each other. Citreicella Mur is very similar (65\% identical) to that of Sinorhizobium meliloti, a ratified manganese-responsive regulator in that species (Platero et al., 2007). Finally, one Roseobacter strain, Octadecabacter antarcticus 307, had two Mur polypeptides; one resembled that in Ruegeria pomeroyi and the other is of the Sulfitobacter/Roseobacter GA101 type. These different sub-types of Mur are seen in a relatedness tree, which also includes Mur polypeptides from other bacteria (Supplementary Figure S1A, B, and C).

The ABC-type transporter SitABCD is also widespread in the Roseobacters, with all but four strains (see below) containing the corresponding four genes, arranged contiguously (Table 2). Closer examination of the sequences of SitA, SitB, SitC and SitD showed that in most strains, these closely resemble $(\sim 70 \%$ identical $)$ those in Ruegeria pomeroyi DSS-3 itself (Table 2). Several other Roseobacters contained a somewhat distinct version of the SitABCD polypeptides, which were very similar in the different strains ( $\sim 77 \%$ identity) but less so ( $40 \%-50 \%$ identity) to those of the Ruegeria pomeroyi group (Table 2). This second sub-class of Roseobacter SitABCD polypeptides closely resembled ( $\sim 75 \%$ identical) those of various strains of Rhizobium, Sinorhizobium and Agrobacterium, and other bacteria, whose function as manganese transporters has been ratified (Diaz-Mireles et al., 2004; Davies and Walker, 2007). Finally, the SitABCD polypeptides of Loktanella vestfoldensis SKA53 are somewhat divergent from those homologues in all the other Roseobacters and more closely resembles deduced SitA-like polypeptides in two other marine bacteria, Halomonas elongata and Pelagibacterium halotolerans.

It was noted that all those strains with a form of the Mur polypeptide that did not closely resemble that of Ruegeria pomeroyi (see above) also had SitABCD polypeptides that were somewhat divergent from those in that species (Table 2). However, some strains with the 'non-Ruegeria pomeroyi' version of SitABCD (for example, Dinoroseobacter shibae) did have the 'conventional' form of Mur that resembles that of Ruegeria pomeroyi (Table 2).

Although the SitA polypeptides may be of different sub-classes, all the corresponding sitA genes are preceded by sequences that strongly resemble MRS motifs, indicating that they are regulated in a similar fashion, via the Mur transcriptional regulator (Supplementary Figure S2).

Two Roseobacter strains, Ruegeria sp. TrichCH4B and Citreicella sp. 357, contain a gene (SCH4B_0376 and C357_18417, respectively) whose product resembles $\mathrm{MntH}$, a very different $\mathrm{Mn}^{2+}$ transporter, being $\sim 50 \%$ ( $E$ value $1 \mathrm{e}^{-120}$ ) identical to $E$. coli $\mathrm{MntH}$. These mntH genes were both preceded by convincing MRSs, strongly suggesting that they, too, are regulated by Mur, in response to $\mathrm{Mn}^{2+}$ availability (Supplementary Figure S2). Ruegeria sp. TrichCH4B contains both MntH and SitABCD, but in Citreicella sp. 357, $\mathrm{MntH}$ is its only recognisable manganese transporter (Table 2). 
MntX of Roseovarius nubinhibens is a Mur-regulated $\mathrm{Mn}^{2+}$ transporter

As mentioned above, four genome-sequenced Roseobacter strains lack any SitABCD homologues. One of these, Citreicella sp 357, contains $\mathrm{MntH}$, but Roseovarius nubinhibens ISM, Oceanicola granulosus HTCC2516 and Rhodobacterales strain HTCC2255 lack any previously defined $\mathrm{Mn}^{2+}$ transporter. However, these strains, and only these three strains, each contain a gene, termed mntX, with two significant features; it is preceded by an MRS motif (Supplementary Figure S2; Rodionov et al., 2006), and likely encodes an inner membrane integral protein, with eight or nine predicted trans-membrane helices as shown by SPLIT 4.0 (Juretic et al., 2002), PREDTMBB (Bagos et al., 2004), pSORTB (Yu et al., 2010) and iMembrane (Kelm et al., 2009) analyses. These MntX polypeptides are $\sim 400$ amino acids in length (for example, 387 for Roseovarius nubinhibens ISM_02005 gene product) and are categorised as 'function unknown', with their C-terminal 300 amino acids corresponding to a domain of unknown function (DUF2899 (pfam11449)) and with a less well-conserved $\mathrm{N}$-terminal region. Thus, $\mathrm{mntX}$ likely encodes a novel manganese transporter that substitutes for SitABCD and/or MntH and is regulated by Mur, in response to Mn availability. (This MntX has no similarity to a hydrophobic Mn exporter, also termed MntX, described in Neisseria meningitides by Veyrier et al. (2011)).

We confirmed these predictions for the mntXcontaining strain, Roseovarius nubinhibens ISM. First, a 1574-bp fragment that included its mntX gene (ISM_02005) plus upstream regulatory sequences was amplified from genomic DNA and cloned into the wide host-range vector pOT2 (Allaway et al., 2001), forming pBIO2066. When mobilised into the Ruegeria pomeroyi SitA ${ }^{-}$mutant J529, this plasmid fully restored the ability to grow on Mn-depleted medium (Figure 2).

To study the regulation of mntX of Roseovarius nubinhibens ISM, we made a lacZ transcriptional fusion. This comprised a 569-bp fragment that spanned its predicted promoter, plus its predicted MRS cis-acting regulatory sequence, and extending into the first $180 \mathrm{bp}$ of the $m n t X$ structural gene, cloned into the promoter-probe plasmid pBIO1628, forming pBIO2067. This plasmid was mobilised by conjugation into wild-type Roseovarius nubinhibens and into wild-type Ruegeria pomeroyi. For both species, the $\beta$-galactosidase activities were 16-fold greater when transconjugants were grown in $\mathrm{Mn}$ depleted than in Mn-replete medium, with, respectively, 1607 and 94 Miller units. To show that this regulation was mediated by Mur, the pBIO2067 fusion was mobilised into the Ruegeria pomeroyi Mur- mutant J528. In this background, the fusion was expressed at high level in both $+\mathrm{Mn}$ and $-\mathrm{Mn}$ media (1861 and 1889 Miller units, respectively). Thus, Mur of one Roseobacter strain can regulate mntX of a different species, consistent with their

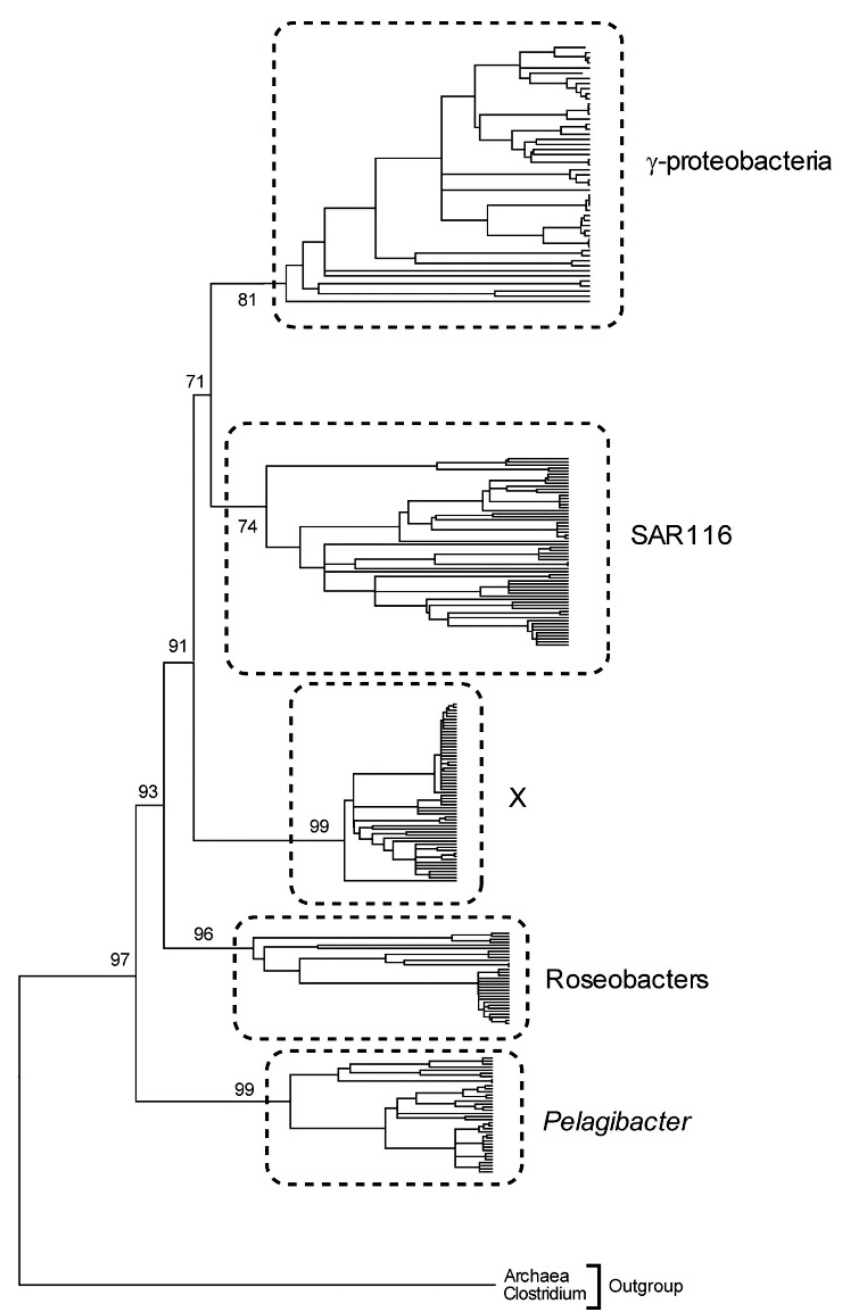

Figure 3 Neighbour-joining tree of MntX polypeptides in known bacteria and in the GOS marine metagenome. Using Mega5, a tree of the MntX homologues in the bacteria described in the text and in the GOS marine metagenome was constructed, bootstrap values being presented. These homologues were of five sub-classes as indicated, each corresponding to those found in the bacterial clade that is shown. Group ' $\mathrm{X}$ ' contains no sequences from any known bacterium. The number of GOS sequences found in each of the five groups are indicated. Accession numbers of the individual GOS sequences are in Supplementary Table S2 and blow-up versions of the five branches are shown in Supplementary Figure S3, A-E.

similar MRS box sequences (Supplementary Figure S2).

MntX in other, important bacterial clades

There are many significant MntX homologues, in addition to those in the three Roseobacter strains, above. These homologues were restricted to a few genera in the Orders Vibrionales, Altermonadales and Oceanospirillales of $\gamma$-proteobacteria and in two $\alpha$-proteobacterial clades, namely SAR11 and SAR116. Significantly, all these taxa are sea-dwelling. These MntX polypeptides fell into four subclasses, each being associated with one of the above bacterial lineages (Figure 3), as described below. 
Vibrionales: Among this Order of marine bacteria, there are genome-sequenced strains in Aliivibrio (A. salmonicida), Photobacterium and Vibrio itself. The single Aliivibrio and three Photobacterium strains contain MntX homologues as do all but four of the 99 sequenced strains of Vibrio itself. The exceptions were strains of Vibrio campbellii, Vibrio caribbenthicus, Vibrio nigripulchritudo and Vibrio sinaloensis, but these contained at least one other known $\mathrm{Mn}^{2+}$ transporter, either SitABCD alone (V. caribbenthicus, $V$. sinaloensis, $V$. nigripulchritudo) or both SitABCD plus MntH (V. campbellii DS40M4). Furthermore, the sitABCD operon of $V$. caribbenthicus and $V$. sinaloensis abuts a mur-like gene whose product resembled those of Citreicella and Sulfitobacter (Supplementary Figure S1). Finally, some Vibrio and Photobacterium strains contain MntH and MntX, but not SitABCD (Supplementary Table S1). (The available Vibrio genomes of all strains with no known Mn transporters were in draft form and were not included).

Altermonadales MntX homologues occur in strains of Colwellia, Alteromonas, Pseudoalteromonas, all of which are Altermonadales $\gamma$-proteobacteria. Here too, there is diversity within a genus; thus, Alteromonas macleodii ATCC 27126 contains mntX but strain 'Deep ecotype' does not (Supplementary Table S1).

The Oceanospirillales also include some strains with mntX (Marinomonas mediterranea MMB-1 and $M$. posidonica IVIA-Po-181), but others in the same genus (MWYL1) lack it (Supplementary Table S1).

Two other important marine $\alpha$-proteobacteria contain mntX genes. These two clades, SAR11 (Ca. Pelagibacter ubique) and SAR116 (Ca. Puniceispirillum marinum) were first detected by cultureindependent analysis of bacterial DNA sequences from the Sargasso Sea (Rappe et al., 2002; Stingl et al., 2007), but have now been grown in pure culture and some have been genome-sequenced.

The SAR11 bacteria are the most abundant on Earth (Morris et al., 2002). Of four sequenced strains, one, Ca. Pelagibacter sp. IMCC9063, encodes a homologue of MntX (SAR11G3_00277), which is 39\% identical to that of Roseovarius nubinhibens. Interestingly, this $m n t X$-like gene is adjacent to a divergently transcribed gene (SAR11G3_00278) whose product is predicted to be in the MntR family, being $50 \%$ identical to the $E$. coli version of this manganeseresponsive transcriptional repressor. Strain IMCC9063 is in Pelagibacter subgroup 3, rather distantly related to other genome-sequenced strains (HTCC7211, HTCC1062 and HTCC1002) (Oh et al., 2011), all of which lack MntX. Indeed, these three strains lack genes for any known $\mathrm{Mn}^{2+}$ transporters.

SAR116 clade bacteria are also widespread throughout the oceans, although not as abundant as the SAR11 group (Morris et al., 2012). The genome-sequenced SAR116 strains, Ca. Puniceispirillum marinum IMCC1322 (Oh et al., 2010) and sp. HIMB100 both contain genes, (SAR116_1166 and HIMB100_00004540, respectively) whose products are $46 \%$ identical to MntX of Roseovarius nubinhibens.

MntX of Ca. Pelagibacter sp. IMCC9063 and Vibrio cholerae O1 biovar El Tor str. N16961 are $\mathrm{Mn}^{2+}$ transporters

We tested directly if two of these predicted $m n t X$ genes encoded functional $\mathrm{Mn}^{2+}$ transporters, again by trying to correct the growth phenotype of the SitA- mutant of Ruegeria pomeroyi. We chose $C a$. Pelagibacter sp. IMCC9063 and V. cholerae, because these are especially important bacteria, although for very different reasons.

For Ca. Pelagibacter sp. IMCC9063, we acquired a 1345-bp fragment containing intact $m n t X$ (SAR11G3_00277), whose codon usage was optimised (using OPTIMIZER (Puigbò et al., 2007)) for expression in the Roseobacters (whose GC content is $\sim 60 \%$, compared with $\sim 30 \%$ in Pelagibacter). This $m n t X$-like gene was sub-cloned into the wide hostrange vector pRK415 (Keen et al., 1988) and the resulting plasmid (pBIO2088) was conjugated into the Ruegeria pomeroyi DSS-3 SitA- mutant J529. It fully corrected the mutant's growth defect in $\mathrm{Mn}$ deficient medium (Figure 2).

Similarly, we created a plasmid, pBIO2150, which contained the mntX gene (VC1688) of V. cholerae $\mathrm{O} 1$ biovar eltor str. N16961 cloned in the wide host-range vector pRK415. When conjugated into the Ruegeria pomeroyi SitA ${ }^{-}$mutant J529, the $V$. cholera mntX gene in this plasmid also fully complemented the mutant's growth defect in Mn-deficient medium (Figure 2).

\section{Distribution of MntX polypeptides in marine metagenomes}

Given that MntX homologues were found in diverse marine bacterial clades, it was of interest to examine their distribution in bacterial metagenomic data sets, most notably those obtained in the Global Ocean Sampling (GOS) expeditions (Rusch et al., 2007; Yooseph et al., 2007). When MntX of Roseovarius nubinhibens was used in BLASTP-based interrogations of these sequence reads, in CAMERA (Sun et al., 2010), we found many homologues (469 with $E$ values $<9.4 \mathrm{e}^{-20}$ ), only $\sim 10$-fold fewer than when RecA of a number of different bacterial taxa were individually used as the probes. These MntXlike polypeptides occurred in nearly all the sampling sites, in the Atlantic, Pacific and Indian Oceans, further emphasising their abundance in marine bacteria (Supplementary Table S2).

Of these 469 sequence reads, 183 had $>230 \mathrm{bp}$ (corresponding to $>59 \%$ of the total MntX polypeptide sequence) of alignment to Roseovarius nubinhibens mnt $X$ and these were included in the neighbourjoining tree of MntX polypeptides (Figure 3; Supplementary Figure S3 A-E). These show that the GOS sequences fall into five different branches of the 
MntX family, four of which mapped onto those represented by the MntX polypeptides of known bacterial strains (see above). Of these four groups, the most populous (59 sequences) was the one that closely resembled the SAR116-type, followed by the Pelagibacter version (35 sequences), then the Roseobacters (27 sequences) and, most infrequently, the $\gamma$ proteobacterial group, with two GOS representatives. Significantly, 59 closely related sequences (Clade X in Figure 3) were in a cluster that did not include any sequences from known organisms, so may originate from an as-yet unknown marine bacterial clade. It was striking that the DNA in the GOS reads that included these Clade X-like mntX genes all had very low ( $\sim 30 \%)$ GC contents, as do the genomes of the SAR11 clade. Although some of these reads included a flanking gene, none of these encoded a taxonomically diagnostic product, so the origin of these $m n t X$ genes is not known.

\section{Discussion}

Despite the importance of metal acquisition by marine bacteria, there have been few genetic experimental studies on this subject, the work on the effects of iron on the transcriptome and proteome of Ca. Pelagibacter ubique by Smith et al. (2010) being a recent exception.

The starting point for our study, on the mechanism and regulation of manganese uptake in a model Roseobacter strain, Ruegeria pomeroyi DSS-3, generated no real surprises. As in other $\alpha$-proteobacteria, notably the rhizobia, this Roseobacter used the ABC-type transporter SitABCD, whose expression was regulated by the Mur transcriptional regulator, which binds to regulatory, MRS, cis-acting sequences, to repress transcription under Mnreplete conditions. This likely applies to most of the other Roseobacters, on the basis of their possession of all these components of the manganese homoeostatic machinery. It was clear, though, that both the SitABCD transporters and the Mur regulators were in different sub-classes in different strains and that, mostly, there was an association between the versions of each of these sets of polypeptides in individual strains; thus, if a given strain had a SitABCD transporter that closely resembled that of (for example) Ruegeria pomeroyi, then so did its Mur regulator, in most cases (see Table 2).

More interesting were those few Roseobacter strains that lacked the SitABCD transporter, leading us to investigate the novel MntX polypeptide. This was first invoked in the Roseobacters as a possible manganese transporter on the basis of circumstantial bioinformatic evidence, as (a) it occurred only in those few Roseobacter strains that lacked SitABCD, (b) the $m n t X$ gene is preceded by a regulatory MRS motif and (c) the MntX polypeptide is predicted to be membrane-bound. We now have direct genetic evidence that the MntX of Roseovarius nubinhibens
ISM is functional and is regulated in response to $\mathrm{Mn}$ availability via the Mur transcriptional regulator. Within the Roseobacter clade, it appears that nearly all strains either contain the SitABCD or the MntX system but not both; a few have the very different, third class of $\mathrm{Mn}^{2+}$ transporter $\mathrm{MntH}$ instead of (in Citreicella sp. 357) or, as well as (Ruegeria sp. Trich $\mathrm{CH} 4 \mathrm{~B})$ one of the other types. In such stains with multiple systems of $\mathrm{Mn}^{2+}$ transport, it will be of interest to determine their relative importance, perhaps under different environmental conditions.

It is now clear that MntX is more widespread in the proteobacteria, in the Oceanospirillales, Altermonadales and Vibrionales in the $\gamma$-subphylum and in the SAR11, SAR116 and Roseobacter clades of $\alpha$ proteobacteria. It is striking that despite their taxonomic diversity, all these Orders are largely or exclusively marine in their habitats. Notably, though, even within a single genus (for example, Marinomonas or Ca. Pelagibacter) some species/ strains harbour mntX and others do not. Although this type of distribution may point to the acquisition of $m n t X$ by horizontal gene transfer, the fact that the MntX's within any given clade (for example, Vibrionales) closely resemble each other compared with those that are in other taxa, suggests that the horizontally acquired gene was stably maintained throughout the evolution of that Order.

Given that cholera is still a major killer, with 3-5 million cases and around 120000 deaths annually worldwide, the finding of the MntX transporter in many species and genera of the Vibrionales, and in $V$. cholerae in particular, may prompt some future work on this system in these important pathogens. A microarray study has shown that the expression of the mntX gene, VC1688, in V. cholerae 0395 was repressed slightly (2.9-fold) in Fe-replete compared with Fe-depleted medium (Mey et al., 2005). However, to our knowledge, there have been no studies on the effects of manganese on its transcription, or, indeed on the role of manganese acquisition on the virulence and/or environmental survival of $V$. cholerae. It will therefore be of interest to examine the effects of mutating this gene on these phenotypes.

MntX occurs in several bacteria (SAR11, SAR116 and Roseobacters) that are abundant in the oceans, so it was not surprising to see large number of homologues in marine metagenomes. MntX homologues in the GOS metagenome are slightly less abundant than those of the SitABCD type and 3.7-fold more common than the MntH homologues in that database. Interestingly, 59 out of $183 \mathrm{MntX}$ polypeptides formed a tightly related cluster that did not include members of any known bacterial species. This cluster was characterised by a very low GC content, as also occurs in the SAR11 genomes. However, we do not know if this cluster represents a novel, unknown clade of bacteria that coincidentally has a high AT:GC ratio or if there are SAR11 strains that contain this form of MntX but whose 
genome sequences are not currently available. We saw no convincing homologues of MntX in any other metagenomic database-from soils, or gut, for example-stressing the association of the MntX polypeptide and marine environments.

Our observations on the distribution of MntX in the various marine bacteria have other implications. Perhaps most significant is that some strains lack any of the known manganese transporters $\mathrm{MntH}$, MntX and SitABCD. Several of these may therefore possess another, as yet unidentified system or else have evolved a biochemistry in which $\mathrm{Mn}$ is required in low amounts, if at all. Given that three of the sequenced strains of the important SAR11 Ca. Pelagibacter ubique are of this type, this topic warrants further investigation.

The fact that mntX seems to be confined to marine bacteria prompts some questions; is it a more effective transporter of the very low concentrations of manganese that prevail in the oceans, and/or does it operate more effectively under saline conditions, as occurs for other systems, such as the betaine/carnitine/choline transporter (see Ziegler et al., 2010)?

Manganese is very scarce in the open oceans, so one might expect the manganese transporters to be expressed constitutively under most natural circumstances. The very fact that the Mn-responsive Mur, plus its cis-acting MRS targets have been retained suggests that a significant proportion of the population of cells must spend sufficient time in naturally occurring Mn-replete conditions to justify the retention over evolutionary time of a negativelyacting regulator that can respond to such nutritional largesse for this metal. Such conditions might apply, for example, if the cells are transiently in the vicinity of a dense plankton bloom or if present in sediments, as recently demonstrated for Roseobacter strains (Lenk et al., 2012). Indeed, this consideration may apply more broadly, encompassing analogous regulatory systems that sense other essential metals, such as molybdenum, nickel or iron, which are also rare in the seas.

\section{Acknowledgements}

This research was supported in part by a Doctoral Training Grant and the Institute Strategic Programme Grant from the BBSRC to the Institute of Food Research. We thank Andrew Curson, Arnoud van Vliet and Matthew Sullivan for helpful discussions.

\section{References}

Allaway D, Schofield NA, Leonard ME, Gilardoni L Finan TM, Poole PS. (2001). Use of differential fluorescence induction and optical trapping to isolate environmentally induced genes. Environ Microbiol 3: 397-406.

Bagos PG, Liakopoulos TD, Spyropoulos IC, Hamodrakas SJ. (2004). A Hidden Markov Model method, capable of predicting and discriminating beta-barrel outer membrane proteins. BMC Bioinform 5: 29.

Baumann P, Baumann L. (1981). The marine Gram-negative eubacteria: genera Photobacterium, Beneckea, Alteromonas, Pseudomonas, and Alcaligenes. In: Starr MP, Stolp H, Trüper HG, Balows A, Schlegel HG (eds) The Prokaryotes, BerlinSpringer-Verlag, pp 1302-1331.

Boyer E, Bergevin I, Malo D, Gros P, Cellier MF. (2002). Acquisition of $\mathrm{Mn}$ (II) in addition to $\mathrm{Fe}(\mathrm{II})$ is required for full virulence of Salmonella enterica serovar Typhimurium. Infect Immun 70: 6032-6042.

Brinkhoff T, Giebel HA, Simon M. (2008). Diversity, ecology, and genomics of the Roseobacter clade: a short overview. Arch Microbiol 189: 531-539.

Landing WM, Bruland KW. (1987). The contrasting biogeochemistry of iron and manganese in the Pacific Ocean. Geochim Cosmochim Acta 51: 29-43.

Buchan A, González JM, Moran MA. (2005). Overview of the marine roseobacter lineage. Appl Environ Microbiol 71: 5665-5677.

Chao TC, Becker A, Buhrmester J, Pühler A, Weidner S. (2004). The Sinorhizobium meliloti fur gene regulates, with dependence on $\mathrm{Mn}(\mathrm{II})$, transcription of the sitABCD operon, encoding a metal-type transporter. $J$ Bacteriol 186: 3609-3620.

Curson ARJ, Todd JD, Sullivan MJ, Johnston AWB. (2011). Catabolism of dimethylsulphoniopropionate: microorganisms, enzymes and genes. Nat Rev Microbiol 9: 849-859.

Davies BW, Walker GC. (2007). Disruption of sitA compromises Sinorhizobium meliloti for manganese uptake required for protection against oxidative stress. J Bacteriol 189: 2101-2109.

Díaz-Mireles E, Wexler M, Sawers G, Bellini D, Todd JD, Johnston AWB. (2004). The Fur-like protein Mur of Rhizobium leguminosarum is a $\mathrm{Mn}(2+)$-responsive transcriptional regulator. Microbiol 150: 1447-1456.

Díaz-Mireles E, Wexler M, Todd JD, Bellini D, Johnston AWB, Sawers RG. (2005). The manganese-responsive repressor Mur of Rhizobium leguminosarum is a member of the Fur-superfamily that recognizes an unusual operator sequence. Microbiol 151: 4071-4078.

Figurski DH, Helinski DR. (1979). Replication of an origincontaining derivative of plasmid RK2 dependent on a plasmid function provided in trans. Proc Natl Acad Sci USA 76: 1648-1652.

González JM, Covert JS, Whitman WB, Henriksen JR, Mayer F, Scharf B et al. (2003). Silicibacter pomeroyi sp. Nov. and Roseovarius nubinhibens sp. Nov., dimethylsulfoniopropionate-demethylating bacteria from marine environments. Int J Syst Evol Microbiol 53: 1261-1269.

González JM, Mayer F, Moran MA, Hodson RE, Whitman WB. (1997). Microbulbifer hydrolyticus gen. Nov., sp. Nov., and Marinobacterium georgiense gen. Nov., sp. Nov., two marine bacteria from a lignin-rich pulp mill waste enrichment community. Int J Syst Bacteriol 47: 369-376.

Hohle TH, Franck WL, Stacey G, O’Brian MR. (2011). Bacterial outer membrane channel for divalent metal ion acquisition. Proc Natl Acad Sci USA 108: 15390-15395.

Johnston AWB, Todd JD, Curson ARJ, Lei S, NikolaidouKatsaridou N, Gelfand MS et al. (2007). Living without Fur: the subtlety and complexity of ironresponsive gene regulation in the symbiotic bacterium Rhizobium and other alpha-proteobacteria. Biometals 20: $501-511$. 
Juretic D, Zoranic L, Zucic D. (2002). Basic charge clusters and predictions of membrane protein topology. J Chem Inf Comput Sci 42: 620-632.

Keen NT, Tamaki S, Kobayashi D, Trollinger D. (1988). Improved broad-host-range plasmids for DNA cloning in gram-negative bacteria. Gene 70: 191-197.

Kelm S, Shi J, Deane CM. (2009). iMembrane: homologybased membrane-insertion of proteins. Bioinfo 25: 1086-1088.

Lee JW, Helmann JD. (2007). Functional specialization within the Fur family of metalloregulators. Biometals 20: 485-499.

Lenk S, Moraru C, Hahnke S, Arnds J, Richter M, Kube M et al. (2012). Roseobacter clade bacteria are abundant in coastal sediments and encode a novel combination of sulfur oxidation genes. The ISME J (e-pub ahead of print 28 June 2012; Doi:10.1038/ismej.2012.66).

Makui H, Roig E, Cole ST, Helmann JD, Gros P, Cellier MF. (2000). Identification of the Escherichia coli K-12 nramp orthologue $(\mathrm{MntH})$ as a selective divalent metal ion transporter. Mol Microbiol 35: 1065-1078.

Menscher EA, Caswell CC, Anderson ES, Roop RM 2nd. (2012). Mur regulates the gene encoding the manganese transporter $\mathrm{MntH}$ in Brucella abortus 2308 J Bacteriol 194: 561-566.

Mey AR, Wyckoff EE, Kanukurthy V, Fisher CR, Payne SM. (2005). Iron and fur regulation in Vibrio cholerae and the role of fur in virulence. Infect Immun 73: 8167-8178.

Middag R, de Baar HJW, Laan P, CaiPH van Ooijen JC. (2011). Dissolved manganese in the Atlantic sector of the Southern Ocean. Deep-Sea Res 58: 2661-2677.

Moran MA, Buchan A, González JM, Heidelberg JF, Whitman WB, Kiene RP et al. (2004). Genome sequence of Silicibacter pomeroyi reveals adaptations to the marine environment. Nature 432: 910-913.

Moran MA, Reisch CR, Kiene RP, Whitman WB. (2012). Genomic insights into bacterial DMSP transformations. Ann Rev Mar Sci 4: 523-542.

Morris RM, Frazar CD, Carlson CA. (2012). Basin-scale patterns in the abundance of SAR11 subclades, marine actinobacteria (om1), members of the Roseobacter clade and ocs116 in the South Atlantic. Environ Microbiol 14: 1133-1144.

Morris RM, Rappe MS, Connon SA, Vergin KL, Siebold WA, Carlson CA et al. (2002). SAR11 clade dominates ocean surface bacterioplankton communities. Nature 420: 806-810.

Oh HM, Kang I, Lee K, Jang Y, Lim SI, Cho JC. (2011). Complete genome sequence of strain IMCC9063, belonging to SAR11 subgroup 3, isolated from the Arctic Ocean. J Bacteriol 193: 3379-3380.

Oh HM, Kwon KK, Kang I, Kang SG, Lee JH, Kim SJ et al. (2010). Complete genome sequence of 'Candidatus Puniceispirillum marinum' IMCC1322, a representative of the SAR116 clade in the alphaproteobacteria. $J$ Bacteriol 192: 3240-3241.

Platero R, de Lorenzo V, Garat B, Fabiano E. (2007). Sinorhizobium meliloti fur-like (Mur) protein binds a fur box-like sequence present in the $m n t A$ promoter in a manganese-responsive manner. Appl Env Microbiol 73: 4832-4838.

Puigbò P, Guzmán E, Romeu A, Garcia-Vallvé S. (2007). OPTIMIZER: a web server for optimizing the codon usage of DNA sequences. Nucl Acid Res 35: W126-131.

Rappe MS, Connon SA, Vergin KL, Giovannoni SJ. (2002). Cultivation of the ubiquitous SAR11 marine bacterioplankton clade. Nature 418: 630-633.
Rodionov DA, Gelfand MS, Todd JD, Curson ARJ Johnston AWB. (2006). Computational reconstruction of iron- and manganese-responsive transcriptional networks in alpha-proteobacteria. PLoS Comput Biol 2: e163.

Rossen L, Shearman CA, Johnston AWB, Downie JA. (1985). The nodD gene of Rhizobium leguminosarum is autoregulatory and in the presence of plant exudate induces the nod A,B,C genes. EMBO J 4: 3369-3373.

Rudolph G, Hennecke H, Fischer HM. (2006). Beyond the Fur paradigm: iron-controlled gene expression in rhizobia. FEMS Microbiol Rev 30: 631-648.

Rusch DB, Halpern AL, Sutton G, Heidelberg KB, Williamson S, Yooseph S et al. (2007). The Sorcerer II global ocean sampling expedition: Northwest Atlantic through Eastern Tropical Pacific. PLoS Biol 5: e77.

Smith DP, Kitner JB, Norbeck AD, Clauss TR, Lipton MS, Schwalbach MS et al. (2010). Transcriptional and translational regulatory responses to iron limitation in the globally distributed marine bacterium Candidatus Pelagibacter ubique. PLoS One 5: e10487.

Stingl U, Tripp HJ, Giovannoni SJ. (2007). Improvements of high-throughput culturing yielded novel SAR11 strains and other abundant marine bacteria from the Oregon coast and the Bermuda Atlantic Time Series study site. ISME J 1: 361-371.

Sun S, Chen J, Li W, Altintas I, Lin A, Peltier S et al. (2010). Community cyberinfrastructure for advanced microbial ecology research and analysis: the CAMERA resource. Nucleic Acids Res 39: D546-D551.

Sunda WG, Huntsman SA. (1994). Interactive effects of external manganese, the toxic metals copper and zinc, and light in controlling cellular manganese and growth in a coastal diatom. Limn Oceanogr 43: 1467-1475.

Tebo BM, Johnson HA, McCarthy JK, Templeton AS. (2005). Geomicrobiology of manganese(II) oxidation. Trends Microbiol 13: 421-428.

Todd JD, Kirkwood M, Newton-Payne S, Johnston AWB. (2011). DddW, a third DMSP lyase in a model roseobacter marine bacterium, Ruegeria pomeroyi DSS-3. ISME J 6: 223-226.

Veyrier FJ, Boneca IG, Cellier MF, Taha MK. (2011). A novel metal transporter mediating manganese export (MntX) regulates the $\mathrm{Mn}$ to $\mathrm{Fe}$ intracellular ratio and Neisseria meningitidis virulence. PLoS Pathog 7: e1002261.

Wexler M, Yeoman KH, Stevens JB, de Luca NG, Sawers G, Johnston AWB. (2001). The Rhizobium leguminosarum tonB gene is required for the uptake of siderophore and haem as sources of iron. Mol Microbiol 41: 801-816.

Wieghardt K. (1989). The active sites in manganesecontaining metalloproteins and inorganic model complexes. Angew Chem 28: 1153-1172.

Wood WB. (1966). Host specificity of DNA produced by Escherichia coli: bacterial mutations affecting the restriction and modification of DNA. J Mol Biol 16: 118-133.

Yamamoto K, Ishihama A, Busby SJ, Grainger DC. (2011). The Escherichia coli K-12 MntR miniregulon includes $d p s$, which encodes the major stationary-phase DNAbinding protein. J Bacteriol 193: 1477-1480.

Yooseph S, Sutton G, Rusch DB, Halpern AL, Williamson SJ, Remington K et al. (2007). The Sorcerer II global ocean sampling expedition: expanding the universe of protein families. PLoS Biol 5: e16.

Yu NY, Wagner JR, Laird MR, Melli G, Rey S, Lo R et al. (2010). Psortb 3.0: improved protein subcellular 
localization prediction with refined localization subcategories and predictive capabilities for all prokaryotes. Bioinformatics 26: 1608-1615.

Zhou D, Hardt WD, Galán JE. (1999). Salmonella typhimurium encodes a putative iron transport system within the centisome 63 pathogenicity island. Infect Immun 67: 1974-1981.

Ziegler C, Bremer E, Krämer E. (2010). The BCCT family of carriers: from physiology to crystal structure. Mol Microbiol 78: 13-34.

Supplementary Information accompanies the paper on The ISME Journal website (http://www.nature.com/ismej) 